\title{
CLOUD MOTIONS ON MARS
}

\author{
W. A. BAUM and L. J. MARTIN \\ Planetary Research Center, Lowell Observatory, Flagstaff, Ariz., U.S.A.
}

\begin{abstract}
A search of several thousand plates in the Lowell Observatory collection yielded 28 groups of plates on which the positions of well-defined transient bright spots (often assumed to be clouds) could be followed on a nearly daily basis. These groups of plates were from 15 different oppositions of Mars, starting from 1907 and ending with 1958. All but two of these spanned four nights or more, and the maximum interval covered was thirty nights. Whether they appeared to show motion or not, the successive positions and shapes of all apparently associated bright spots or clouds were plotted on Mercator projections with the use of a projection plate reader especially designed at the Planetary Research Center for planet image studies of this kind. Clouds near the limb were avoided.

The 28 groups of plates yielded 95 cloud histories. More than half appeared to be relatively stationary. Others showed definite motion well in excess of observational error but sometimes followed paths that partly doubled back upon themselves. The mean velocity for non-stationary clouds was found to be $5.6 \mathrm{~km}$ per hour, and the most commonly occurring direction of motion was eastward, particularly at high latitudes. The range of velocities found by this mapping procedure is nearly an order of magnitude smaller than values that have been estimated earlier by others from visual observations. These earlier observations are evidently in error, unless there exist clouds at high elevation, visible only on the limb, that can move much faster than those that were mapped from this photographic survey. More clouds were found in the northern hemisphere than in the southern, and there seemed to be an avoidance of the relatively darker areas of the Martian surface. Certain regions seem to be more favored than others. A few recurrences at identical positions suggest the existence of related topographic features.
\end{abstract}

\section{Introduction}

The title of this paper may turn out to be a misnomer, because the objects we have studied may not necessarily be clouds of suspended particles in the Martian atmosphere. It is not the purpose of this paper to attempt making any distinction between true clouds and surface phenomena, and it is entirely possible that our various cases include both. We have applied the term 'cloud' to any distinct transient local brightening that is not a normal tone of the surface feature in the area where it occurs. Many of these transient bright spots show no detectable movement at all, particularly in such well known areas as Hellas, Elysium, Tharsis, and Amazonis. On the other hand, the relatively stationary brightenings do not always occur in exactly the same positions and configurations.

The Lowell Observatory plate collection includes about 5000 plates of Mars covering a 60-yr period through 1965. More recent observations are being made on cine film and will be studied separately. A search was made through the plate collection to find cases in which the local positions of well-defined clouds could be followed on a nearly daily basis for significant lengths of time. This search yielded 28 groups of plates from fifteen different oppositions of Mars starting with 1907 and ending with 1958. Further work remains to be done on 1956, 1961, 1963, and 1965. All but two of the 28 selected cases spanned four nights or more, and the maximum interval covered was 30 nights. 
This study of the Martian clouds was limited to the smaller and more discrete types, because those covering broader areas are more difficult to map to the accuracy needed for comparing the positions and configurations seen on various plates. The study was also limited to clouds well within the visible disk so as to maintain a high order of accuracy. Limb clouds were avoided, because their positions cannot be determined with sufficient accuracy. Indeed, our inferred velocities differ by an order of magnitude from those that have been estimated earlier by others on the basis of limb cloud observations.

\section{Procedure}

The first step was a plate-by-plate search for those Mars plates which had clouds that could be mapped. Small sketch maps showing the approximate configurations and locations of all clouds were made for each plate. All readable plates were included, regardless of the filter color. The sketch maps were then reviewed to determine which plates showed clouds that might be related to clouds seen on other plates within a reasonable time span. All possible relationships were included, regardless of the motion or non-motion that appeared to be indicated.

A specially designed optical projector was used for measuring cloud positions on the images. Each projected Mars image was adjusted in size, orientation, and position to fit a transparent coordinate graticule superimposed on the projection screen. This graticule was an orthographic projection with latitude and longitude lines at tendegree intervals. Different orthographic graticules were used to accommodate different tilt angles of the Martian axis in two-degree steps with respect to the line of sight.

The clouds were first outlined with a grease-pencil on a transparent plastic sheet covering the projection screen. This outline was then transferred to a Mercator projection by drawing it on a transparent plastic sheet covering a sliding Mercator graticule on top of a Mercator map of the Martian surface. The sliding Mercator graticule, with ten-degree intervals in both coordinates, was placed so that the location of its central meridian on the map corresponded with the central meridian in the photograph. Thus the Mercator projection lines corresponded with the orthographic projection lines.

Final maps were compiled from groupings of these plastic overlays by plotting together the successive positions of apparently related clouds. All together, 29 such maps were prepared, one for each of 28 selected groupings of overlays, except for one case that required two maps to represent the situation completely. When several plates taken during the same day showed the same cloud in about the same position, these cloud outlines were averaged in position and size to show a single symbol for that date. Clouds that could not be related to the others were usually omitted.

\section{Cloud Maps}

Sample maps are shown in Figures 1 and 2. Each covers only a part of Mars. The tendegree intervals of the Mercator projection are equivalent to about $590 \mathrm{~km}$ at the equator. As observed from the earth, such an interval subtends between 1 and 2 sec 


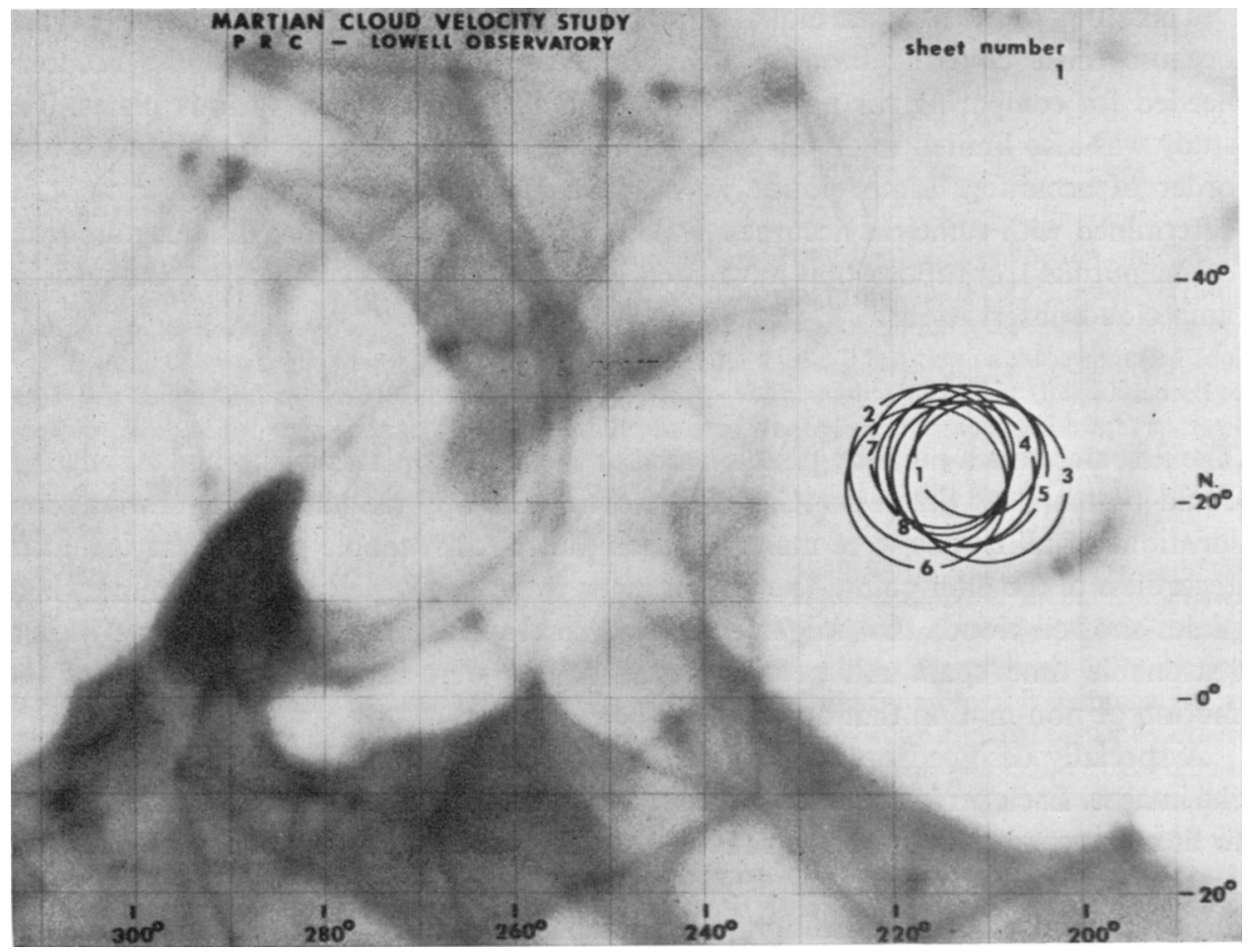

Fig. 1. A typical stationary bright spot in Elysium as it appeared on 33 photographs taken in 1907 (11 July through 18 July). The scatter in the positions of the circles (one for each night) is believed to represent our mapping accuracy.

of arc during the months close to a Martian opposition. We would not be able to say that any of our clouds are smaller than $300 \mathrm{~km}$, although they may in fact be. Like the examples shown in Figures 1 and 2, the maps have all been oriented with north at the top and areographic east at the right. Longitude is numbered from 0 to 360 degrees in a westerly direction. Since Mercator projections were used, azimuths may be drawn as straight lines. The base map of the Martian surface is taken from the NASA-Air Force chart 'Mariner 69 Mars Chart (MEC-2)' drawn under an ACIC contract at the Lowell Observatory. The projection lines at ten-degree intervals have been included from this map, but feature names have been omitted.

Each different number on a symbol represents a different Martian day in chronological order. These days are often consecutive but not always. Gaps between dates arise, either because plates were lacking or because the available plates were not of readable quality. There was no clear-cut case of a cloud totally disappearing one day and reappearing the next. The lower-case letters are an aid in referencing groups or clusters of symbols. The grouping of symbols and the assigning of letters to these groups have necessarily been somewhat arbitrary.

Although a few cases may be ambiguous, most of the transient bright spots or clouds on our maps can be divided into two groups: moving and non-moving. Figure 1 


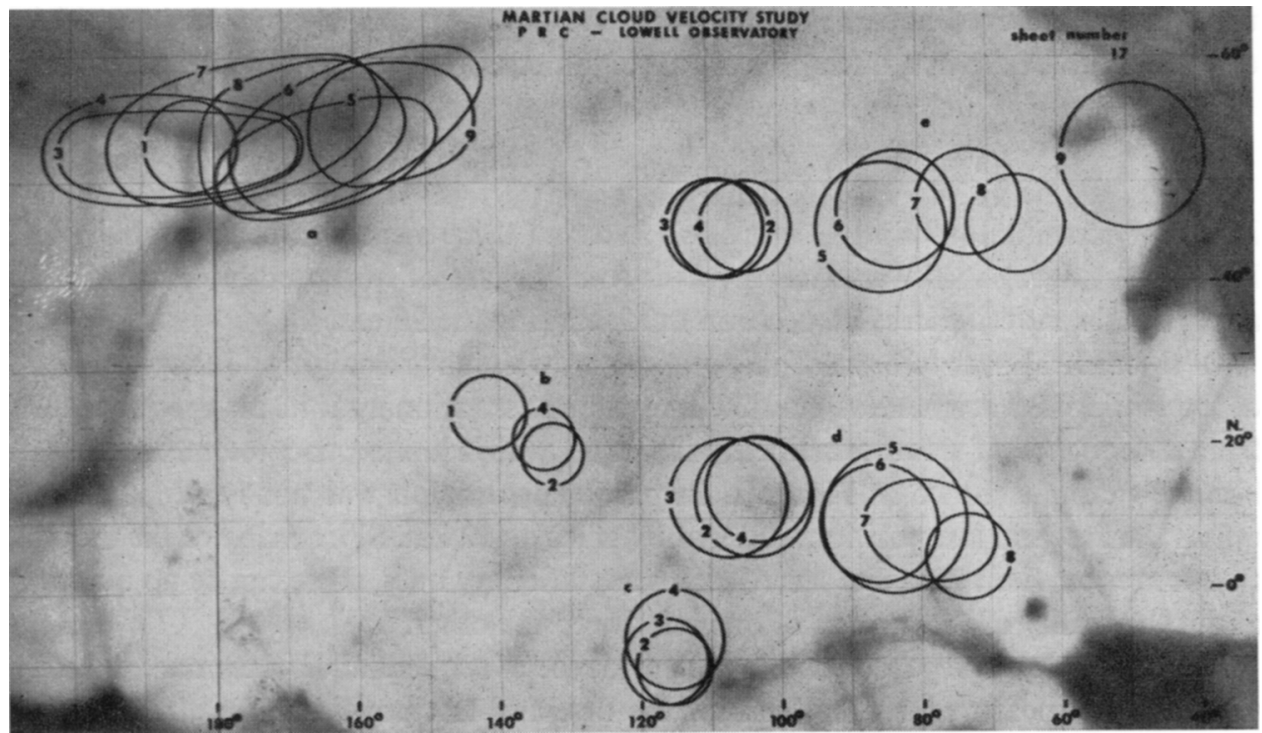

Fig. 2. An array of bright spots or clouds showing various degrees of sequential displacement, as they appeared on 19 photographs taken in 1937 (1 June through 13 June). Rates of motion inferred from the two cases shown top-left and top-right are about $3 \mathrm{~km}$ per hour and $9 \mathrm{~km}$ per hour, respectively.

shows an apparently non-moving local brightening in Elysium as it appeared in 1907. Insofar as we can tell, the scatter in the positions of the circles in Figure 1 is merely a representation of our mapping accuracy. Any progressive motion during the eight-day period covered by these observations was less than $1 \mathrm{~km}$ per hour, although brief random motions could conceivably have exceeded that and remained undetected.

Figure 2 shows an array of transient brightenings having various degrees of motion and non-motion. In the lower half of the figure is the well known 'W-cloud' region where transient brightenings of this kind frequently occur. It is very interesting to compare the appearance of this region in various months and years. For example, the bright spot at about $10^{\circ} \mathrm{S}$ latitude and $120^{\circ} \mathrm{W}$ longitude (scantily documented in this particular case) tends to be stationary and to reappear frequently at the same place. Other parts of the configuration in the lower half of Figure 2 tend to vary much more in position and apparent motion. Stationary clouds and moving clouds are in fact found to occur at exactly the same places at different times.

Figure 2 is of interest primarily because it includes two good illustrations of progressive eastward displacement at about $50^{\circ} \mathrm{N}$ latitude. The overlaid oval shapes at the left side of Figure 2, in the Propontis region, are especially unambiguous. However, this cloud and the one at the right of it both illustrate the jerky manner in which such clouds appear to move. To some degree, they act as though they jump from one favored spot to another, suggestive of terrain relationships. There are also cases in which such motion seems to double back upon itself, or to split into two or more spots in nearby positions. Observations now being obtained on a much more continuous schedule 
under a cooperative Planetary Patrol Program involving six observatories should be of considerable help in reducing future ambiguities of the kind just described.

\section{Summary of Results}

From an examination of all 29 maps, we find that there appear to be 95 usable cloud histories. Although this selection involves some degree of interpretation, we tried to apply consistent criteria of association in identifying the 95 cases.

Of these, 52 appear to be relatively stationary, while the remaining 43 show evidence of sequential displacement. A cloud was regarded as stationary if its mapped locations from a sequence of dates remained unchanged within the accuacy of measurement, like the example shown in Figure 1. In particular, motion was not regarded as real unless the first and last positions differed by at least $400 \mathrm{~km}$. Since many of the moving clouds are found on the same sets of plates as stationary ones, there can be no question about the reality of the motion of one relative to the other.

For the 43 clouds regarded as showing evidence of sequential displacement, velocity vectors were measured on the basis of the first and last positions. As already mentioned, such clouds tend to move jerkily. In some instances they also double partly

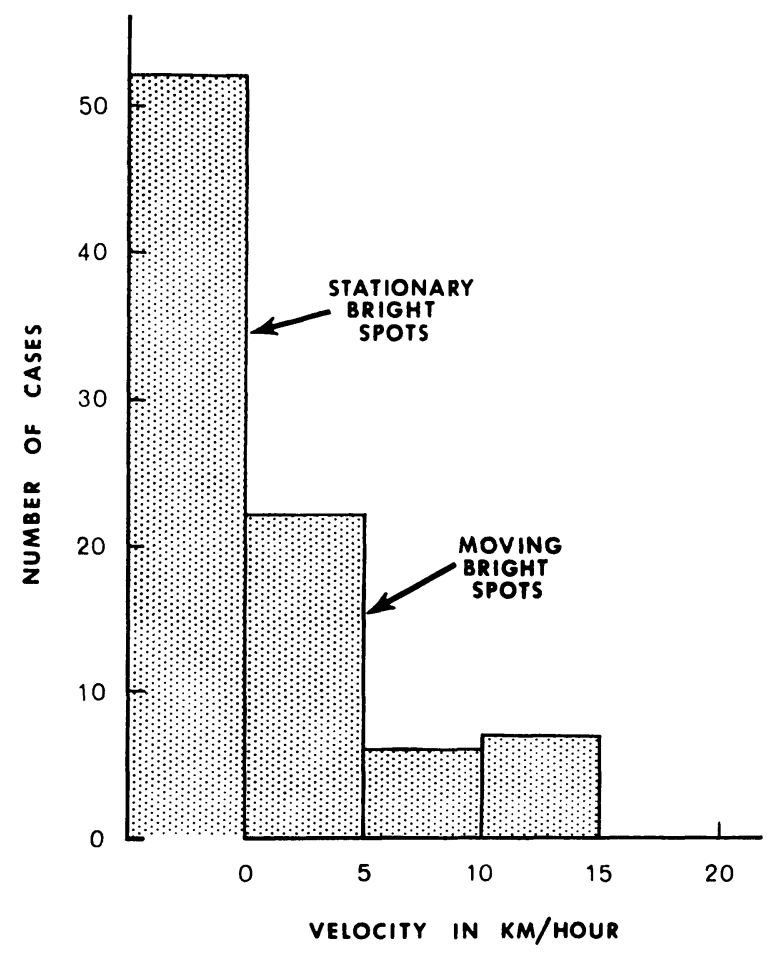

Fig. 3. Distribution of Martian 'cloud' velocities inferred from mapped displacements based on sequences of photographs in the Lowell Observatory plate collection, 1907-1958. This sample was limited to clouds observed over a span of at least four nights. 
back upon their tracks, so that the net displacement between the first and last positions can be less than the total excursion.

Since velocities based on the first and last positions of a cloud are vulnerable to mapping errors unless the time span of observation was long enough, we decided to confine velocity analysis to 35 cases (among the total 43 ) for which observations spanned at least four nights. These 35 , taken together with the 52 clouds regarded as stationary, provide a high-accuracy sample of 87 clouds that yield the histogram in Figure 3. The mean velocity of all 87 clouds is $2.25 \mathrm{~km}$ per hour. The mean velocity for the 35 non-stationary cases alone is $5.60 \mathrm{~km}$ per hour.

The histogram in Figure 4, which includes all 95 original cases, shows that the transient bright spots or clouds did not occur randomly over the whole planet. Only about

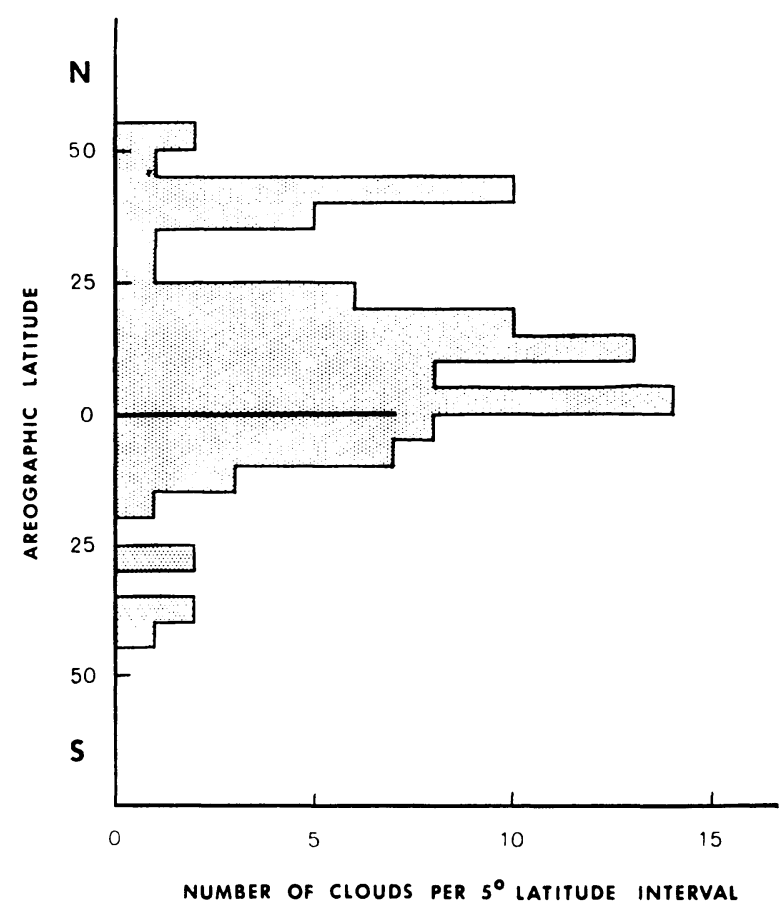

Fig. 4. Latitude distribution of the same Martian 'clouds' that yielded Figure 3, except that cases observed only two nights were included.

one-fourth of them occurred in the southern hemisphere. Almost half of the clouds are found in a relatively narrow belt between the equator and $20^{\circ} \mathrm{N}$ latitude. This result differs from Wells' (1967) summary of earlier cloud sightings that probably contained a stronger seasonal selection effect. In Figure 5 the locations of our clouds are mapped, the dots representing cloud positions on individual nights. It is interesting to note that there seems to be a partial avoidance of the darker areas on Mars. The latitude effects in Figure 4 could, of course, be merely a manifestation of this dark-area avoidance, 
W. A. BAUM AND L. J. MARTIN

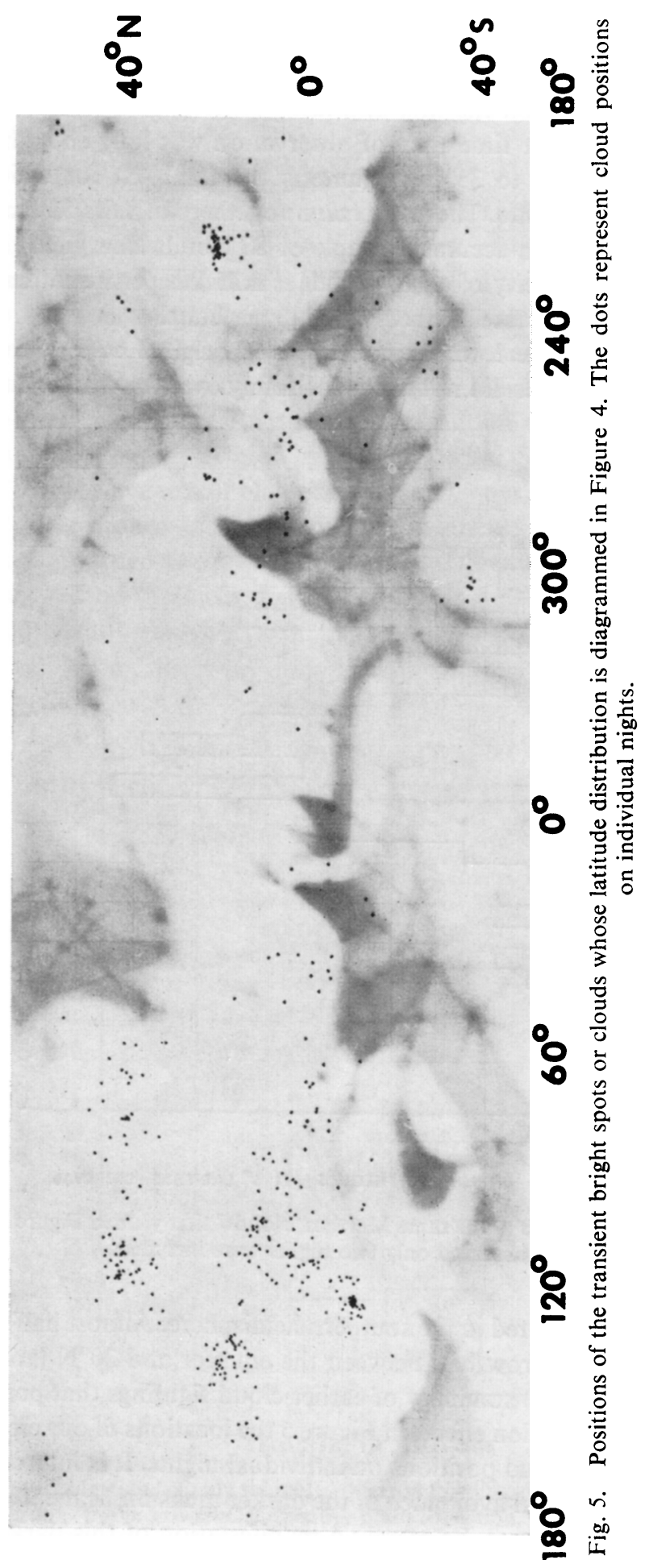


because a large percentage of the dark area of Mars lies in the southern hemisphere. This apparent avoidance of dark areas cannot be an observational selection effect, because the visibility of bright clouds will be greater - not less - over dark areas than over light areas.

Figure 6 shows that somewhat more clouds move eastward and westward than northward and southward. This diagram represents the number of cases falling within each 45-degree sector of the compass. The sample was limited to the 35 non-stationary clouds observed over a time span of four nights or more. On average, those moving eastward tend to have the higher velocities and to occur farther from the equator than those moving in other directions. The sample is not yet large enough to relate these motions clearly to an atmospheric circulation pattern.

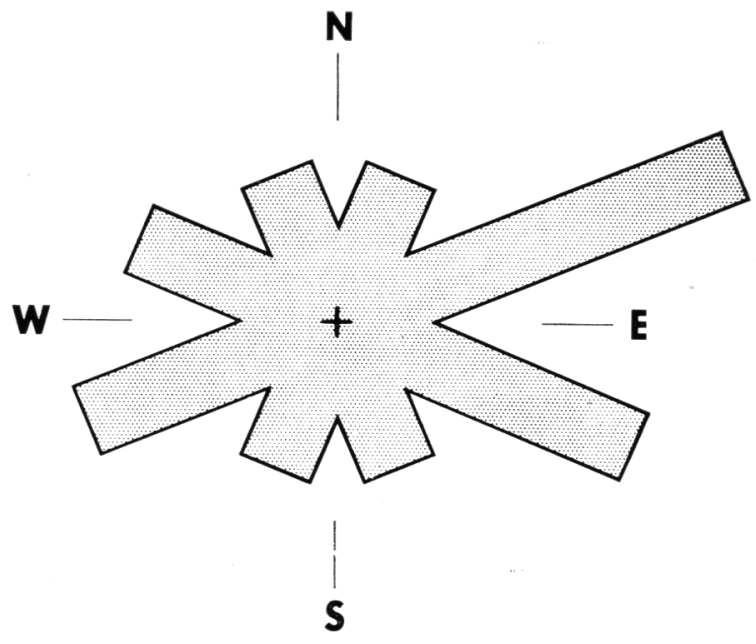

Fig. 6. Directional distribution of Martian 'cloud' motions. This sample includes only the 35 cases for which photographic observations spanned at least four nights and which moved more than $400 \mathrm{~km}$.

Previous observations of Martian cloud movement were reviewed by Gifford (1964). Owing to the comprehensive coverage of Gifford's fine review, we do not present a bibliography of earlier work here. Gifford tabulated 36 velocities and directions of motion that had been reported by various observers since 1873. Many were derived from visual observations, and 20 of the 36 were based on the appearance of clouds at the limb or terminator. The mean of Gifford's tabulated velocities is $36 \mathrm{~km}$ per hour, which is many times larger than the mean of our values derived from the actual mapping of progressive positions in the present study. The highest single value in Gifford's list is $124 \mathrm{~km}$ per hour, credited to the observatoires Jarry-Desloges, and it is more than eight times larger than the highest velocity found in any of our 95 mapped cases. It now seems likely that these earlier observations are largely in error, unless there exists a faster moving class of Martian clouds, possibly at high elevation, that can be seen only when on the limb or terminator. It was because of the large 
uncertainty associated with limb or terminator observations that those regions were intentionally avoided in the present study.

\section{Acknowledgments}

Our work was carried out with the joint support of the Jet Propulsion Laboratory and of NASA Headquarters, and we gratefully acknowledge both. All 29 of our cloud-history maps are contained in a contract report submitted to the Jet Propulsion Laboratory (Martin and Baum, 1969).

\section{References}

Gifford, F. A., Jr.: 1964, Monthly Weather Rev. 92, 435.

Martin, L. J. and Baum, W. A.: 1969, Final Report B, under JPL Contract 951547, August. Wells, R. A.: 1967, Astrophys. J. 147, 1181. 